\title{
Parasite-targeting compound sets identified and ready to go
}

Compounds targeting the three kinetoplastid parasites most relevant to human disease - Leishmania donovani, Trypanosoma cruzi and Trypanosoma brucei (which cause visceral leishmaniasis, Chagas disease and sleeping sickness, respectively) - have been identified in parallel whole-cell phenotypic assays. The results of this research are publicly available, as are physical samples of the compounds.

The GlaxoSmithKline (GSK) diversity set, which consists of 1.8 million compounds, was first screened for molecules that could inhibit parasite growth in axenic

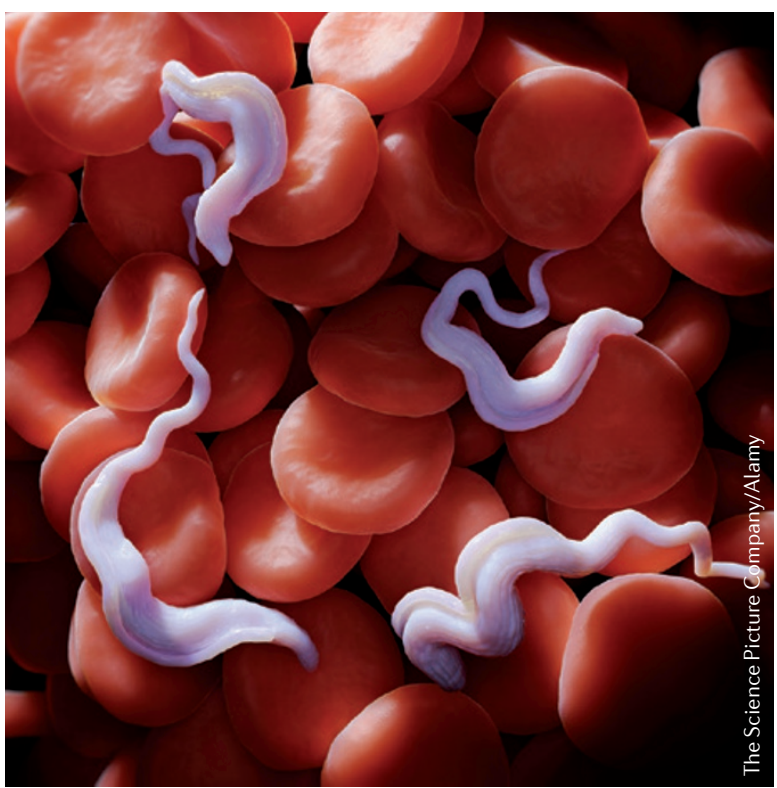

cultures (L. donovani and T. brucei) or in infected mammalian cells (T. cruzi). The results were confirmed in secondary assays with different readouts in order to validate bona fide inhibitors. Importantly, for L. donovani, the secondary screen also switched from an axenic culture to infected mammalian cells. Hits from the three screens were filtered according to physicochemical properties, and approximately 4,000 compounds from each of the three screens were examined in dose-response and mammalian cytotoxicity experiments.

Of these groups of compounds, the most potent, specific and noncytotoxic compounds with suitable physicochemical properties were further refined to generate representative chemical 'boxes', with a maximum of two compounds per chemical cluster per box. Of the 192 anti-L. donovani, 222 anti-T. cruzi and 192 anti-T. brucei compounds, only one compound was common to all three boxes, and fewer than 20 were found in two of the three boxes, suggesting that the vast majority of these compounds target mechanisms specific to each parasite.

The mode of action of each of these compounds was hypothesized from historical GSK screening data and bioinformatics analysis of the three parasite genomes. Many of these compounds probably target serine/threonine or lipid kinases, as known hinge-binding motifs, such as those found in pyrimidines, pyrrolopyrimidines, azaindoles and benzimidazoles, were present in all three boxes. Phosphodiesterases, cysteine peptidases and cytochromes are also likely targets. Indeed, $42 \%$ of the compounds in the T. cruzi box putatively affect the cytochrome sterol 14- $\alpha$ demethylase (CYP51); interestingly, several compounds targeting CYP51 are already in development to treat T. cruzi infections. Potential metal-binding chemicals, methylene dioxy-containing compounds, and molecules with nitro-substituted aryl groups were also overrepresented in these boxes. Consistent with this last observation, nitrosubstituted aromatics are used clinically to treat T. cruzi infections, as these compounds are converted into toxic metabolites by a T. cruzi nitroreductase.

The study - which was a collaborative public-private partnership within an open innovation framework - as well as the physical samples of these boxes, provide an open resource for the global scientific community to develop new therapeutics against these devastating diseases.

Megan Cully

ORIGINAL RESEARCH PAPER Peña, l. et al.

New compound set identified from high throughpu phenotypic screening against three kinetoplastid parasites: an open resource. Sci. Rep. 5, 8771 (2015) 